\title{
Effects of nonselective and selective cyclooxygenase inhibitors on the contractions of isolated bronchial smooth muscle in the horse
}

\author{
Alessandro Menozzi ${ }^{1}$, Cristina Pozzoli ${ }^{2}$, Enzo Poli ${ }^{2}$, Lucia Tagliaferri ${ }^{1}$, \\ Giuseppe Placenza ${ }^{1}$, Simone Bertini ${ }^{1}$
}

University of Parma, ${ }^{1}$ Department of Veterinary Science, ${ }^{2}$ Department of Medicine and Surgery, Parma, Italy

Received February 1, 2018

Accepted May 15, 2018

\begin{abstract}
We evaluated the effects of nonselective cyclooxygenase (COX)-1/COX-2 inhibitors (acetylsalicylic acid, indomethacin, ibuprofen, flunixin meglumine, phenylbutazone), preferential COX-2 inhibitors (diclofenac, meloxicam, carprofen), selective COX-1 inhibitor (SC-560), and selective COX-2 inhibitors (celecoxib, firocoxib, parecoxib) on the contractions of isolated bronchi induced by electrical field stimulation (EFS). Bronchial rings, obtained from lungs of slaughtered horses, were put in isolated organ baths, and the mechanical activity was measured by means of isotonic transducers. Electrical Field Stimulation was applied to the preparations, and the effects of drugs on the amplitude of evoked contractions were measured. Nonselective COX inhibitors did not modify EFS-induced contractions to a relevant degree, except indomethacin which caused a concentration-dependent decrease of the contraction amplitude. Conversely, preferential COX-2 inhibitors enhanced the contractions in a concentration-related fashion, whilst the selective COX-1 inhibitor reduced them. Among selective COX-2 inhibitors, parecoxib increased EFS-evoked contractions whereas celecoxib and firocoxib were ineffective. These results suggest that the inhibition of prostanoid synthesis does not modify the electrical field-stimulated contractions of isolated horse bronchi. Since EFS-induced contractions of horse bronchi were previously shown to be of full cholinergic nature, the increase caused by diclofenac, meloxicam, carprofen, and parecoxib could be due to an inhibition of acetylcholinesterase; in accordance, these drugs potentiated exogenous acetylcholine-induced but not carbachol-induced bronchial contraction. Indomethacin and SC560 might instead decrease bronchial contractions by inhibiting calcium currents. Clinical use of meloxicam and carprofen in horses with bronchial hyper-responsiveness requires caution for a potential risk of causing adverse effects due to bronchoconstriction.
\end{abstract}

COX-1, COX-2, NSAIDs, equine, bronchi

Nonsteroidal anti-inflammatory drugs (NSAIDs) are widely used in clinical practice for their anti-inflammatory, analgesic and antipyretic properties both in humans and animals. Nonsteroidal anti-inflammatory drugs are enzymatic inhibitors which target cyclooxygenase (COX), and thus prevent the synthesis of prostaglandins and thromboxanes. However, the depletion of prostanoids reduces not only their pro-inflammatory effects but also their protective ones, and this is recognised as the main cause of the well-known adverse effects of these drugs.

The breakthrough discovery of two distinct COX isoforms (COX-1 and COX-2) (Xie et al. 1991) propelled the development of novel drugs which, by selectively binding to the inducible enzyme, COX-2, would have been able to block the noxious effects of prostaglandins, while sparing the beneficial ones, unlike traditional NSAIDs, which inhibit both COX isoforms. Much of the initial enthusiasm for these "safer" NSAIDs has been extinguished by further studies, though, which revealed that, like COX-1, COX-2 is also important for the maintenance of homeostasis in several tissues (Kargman et al. 1996;

Address for correspondence:

Prof. Alessandro Menozzi, D.V.M., Ph.D.

Department of Veterinary Science

University of Parma

Strada del Taglio 10, 43126 Parma, Italy

Phone: +390521032797

E-mail: alessandro.menozzi@unipr.it

http://actavet.vfu.cz/ 
Zimmermann et al. 1998). As a matter of fact, only very few COX-2 selective NSAIDs are presently approved for use in humans, and two of them, rofecoxib and valdecoxib, were quickly withdrawn from the market due to the concern for an increased risk of causing serious cardiovascular adverse effects (Bombardier et al. 2000; Atukorala et al. 2013).

Nonselective COX inhibitors such as phenylbutazone, flunixin meglumine, ketoprofen, or acetylsalicylic acid (ASA) are administered to horses for the treatment of musculoskeletal lesions, osteoarthritis or after surgery, and are known to cause adverse effects which include gastrointestinal ulcers, colitis, and bowel motility disorders (MacKay et al. 1983; MacAllister et al. 1993; Dabareiner et al. 1995). Preferential COX-2 inhibitors meloxicam and carprofen, and selective COX-2 inhibitor firocoxib, are also registered for horses as analgesic and anti-inflammatory agents. Although it is known that prostanoids modify the bronchial tone in several species (Horton et al. 1965; Gardiner 1975; Hamberg et al. 1975), COX inhibitors seem mostly devoid of major effects on the contraction of bronchial muscle in vitro (Douglas et al. 1987). However, NSAIDs were found to potentiate the contraction of isolated trachea induced by agonists in the guinea pig and dog (Orehek et al. 1973; McGrogan et al. 1996); and there is abundant evidence (Steinhoff et al. 2014) that ASA and other NSAIDs may be responsible for bronchospasm in human patients, or for exacerbating the clinical signs of asthma (Bianco et al. 1985; Looney et al. 2005; Parker et al. 2016). It has been suggested that NSAIDs could increase bronchial contractions by either reducing the production of myorelaxant prostaglandin $\mathrm{E}_{2}\left(\mathrm{PGE}_{2}\right)$, which is synthesized in response to bronchoconstriction caused by disease or drugs (Smith et al. 1976), or by shifting the conversion of arachidonic acid towards the 5-lypooxygenase-dependent production of leukotrienes, which are potent smooth muscle spasmogenic agents (Christie et al. 1991). Since bronchial $\mathrm{PGE}_{2}$ seems to be mainly produced by COX-1 (Harrington et al. 2008), COX-2 selective inhibitors are indeed thought to be safer than traditional NSAIDs in asthmatic patients. It is possible that $\mathrm{PGE}_{2}$ could play a protective role also in horse bronchi, since for instance a decrease in the synthesis of this prostanoid in horses with recurrent airway obstruction (RAO) was detected (Gray et al. 1992).

The aim of the present study was to assess the effects of nonselective COX-1/COX 2 inhibitors (ASA, indomethacin, ibuprofen, flunixin meglumine, phenylbutazone), preferential COX2 inhibitors (diclofenac, meloxicam, carprofen) and selective COX-1 (SC-560) or COX-2 (celecoxib, firocoxib, parecoxib) inhibitors (Futaki et al. 1994; Brideau et al. 2001), on the contraction of isolated bronchial smooth muscle of the horse, as so far there has been a lack of knowledge regarding the ability of NSAIDs to modify the motility of equine respiratory tract.

\section{Materials and Methods}

Samples of lung were collected from 46 male horses (2-8 years of age), slaughtered at a private abattoir; the experiments were performed between September 2016 and August 2017. The samples were excised from the apical lobes of lungs and were stored in ice-cooled modified Krebs-Henseleit solution of the following composition (mM): $\mathrm{NaCl} 113.0, \mathrm{KCl} 4.7, \mathrm{MgSO}_{4} \cdot 7 \mathrm{H}_{2} \mathrm{O} 1.2, \mathrm{CaCl}_{2} \cdot 2 \mathrm{H}_{2} \mathrm{O} 1.8, \mathrm{KH}_{2} \mathrm{PO}_{4} 1.2, \mathrm{NaHCO}_{3} 25.0$ and dextrose 11.2, for the 10-min transport from the slaughterhouse to the laboratory. Bronchial segments were then isolated from the surrounding parenchyma, and bronchial rings of $0.7-1 \mathrm{~cm}$ diameter, without epithelium, (12 from each horse) were obtained to be used in motility experiments. Each ring was put into a $10 \mathrm{ml}$ organ bath at $37{ }^{\circ} \mathrm{C}$, containing the solution above described, continuously bubbled with $95 \% \mathrm{O}_{2}$ and $5 \% \mathrm{CO}_{2}$ ( $\mathrm{pH}$ 7.4). The bronchial rings were left to stabilize for $60 \mathrm{~min}$ and then the mechanical activity was measured by means of an isotonic transducer (Basile, Milan, Italy) connected to the preparation, developing a passive stretch of $1 \mathrm{~g}$ throughout the entire experiment. Pilot experiments were performed to establish the optimum load to get the best contractile activity. The viability of bronchial rings was assessed by the ability of acetylcholine (ACh) $\left(10^{-7} \mathrm{M}\right)$ to evoke a contractile response $(>0.1 \mathrm{~cm}$ shortening). Electrical Field Stimulation (EFS) was applied by means of two coaxial platinum electrodes positioned $10 \mathrm{~mm}$ from the longitudinal axis of the preparation and used to deliver trains of square wave pulses ( $1 \mathrm{~ms}$ duration, $50 \mathrm{~V}$ amplitude) every $90 \mathrm{~s}$ to the tissue, at the 
frequency of $15 \mathrm{~Hz}$. For each experiment, the intensity was adjusted to a level giving $70-80 \%$ of the maximum tissue response (usually 250-300 mA), in order to be able to measure either an increase or a decrease of the contraction amplitude. Regular phasic contractions were obtained, and the effects of drugs were measured as the percentage of variation of pre-drug amplitude of contractions, assumed as $100 \%$. In a separate set of experiments, concentration-response curves of ACh or nonselective muscarinic agonist, carbachol, were constructed in absence and after $30 \mathrm{~min}$ of incubation with a single aliquot of $10^{-5} \mathrm{M}$ diclofenac, meloxicam, carprofen, or parecoxib. The potency of the agonists was expressed by the concentration giving $50 \%$ of maximum effect $\left(\mathrm{EC}_{50}\right)$ from individual concentration-response curves, and expressed with - $\log \mathrm{EC}_{50}$.

The effects of NSAIDs on the basal tone of horse bronchi was also assessed, by adding the drugs in the organ baths at increasing concentrations, without applying EFS. Moreover, in order to detect a possible relaxant activity by NSAIDs, their effects on the sustained contraction induced by carbachol, a nonselective muscarinic agonist, were studied. For this purpose, a single concentration $\left(10^{-5} \mathrm{M}\right)$ of carbachol was added to the organ bath solution, and the effect was observed until a constant plateau of contraction was achieved; increasing concentrations of the COX inhibitors were then added into the bath solution, and the effect on the amplitude of contraction plateau was measured. For the generation of all concentration-response curves, drugs were added cumulatively into the bath solution in 1-log unit increments of concentration. Each concentration-response curve was fitted to nonlinear regression with variable slope using a commercial software (GraphPad Prism ver. 6.05, GraphPad Inc., San Diego, CA, USA).

\section{Drugs}

Acetylsalicylic acid, indomethacin, ibuprofen, flunixin meglumine, phenylbutazone, diclofenac, meloxicam, carprofen, SC-560, celecoxib, firocoxib, and parecoxib were purchased by Sigma-Aldrich (Sigma Chemical Co., St Louis, MO, USA). Flunixin meglumine was dissolved and diluted to the final concentration in distilled water; all other drugs were dissolved and diluted to the final concentration in 1\% dimethyl sulphoxide (DMSO), and then diluted to the final concentration with distilled water. Such concentration of DMSO was previously tested and did not modify the contractility of preparations or the effect of drugs. All solutions were freshly prepared before each experiment and proper aliquots $(10$ to $100 \mu \mathrm{l})$ were added to the organ baths to achieve the desired molarity.

\section{Statistical analysis}

All data were expressed as means \pm SEM from eight experiments. Differences among data were evaluated by means of unpaired parametric Student's $t$-test. A $P$ value $<0.05$ was considered significant. All calculations were performed using GraphPad Prism software.

\section{Results}

Nonselective COX-1/COX-2 inhibitors, ASA, phenylbutazone, and ibuprofen $\left(10^{-8}-10^{-4} \mathrm{M}\right)$, did not modify the amplitude of EFS-evoked contractions, whereas flunixin meglumine induced only a mild increase of the contractions at $10^{-7} \mathrm{M}-10^{-5} \mathrm{M}$ (Fig. 1A). Conversely, indomethacin reduced the amplitude of EFS-induced contractions in a concentration-dependent manner, reaching a maximal effect of $-30.4 \pm 12.2 \%$ at $10^{-4} \mathrm{M}$ (Fig. 1A).

Preferential COX-2 inhibitor, diclofenac, enhanced EFS-induced contractions in a concentration-related fashion, with a maximal effect of $+19.8 \pm 3.2 \%$ at $10^{-6} \mathrm{M}$, whereas meloxicam and carprofen increased the amplitude of contractions at the higher concentrations only (Fig. 1B). The maximum effects at the concentration of $10^{-4} \mathrm{M}$ were $+28.9 \pm 8.15 \%$ and $+29.2 \pm 9.8 \%$ for meloxicam and carprofen, respectively.

Selective COX-1 inhibitor, SC-560, induced a concentration-dependent decrease of the contraction amplitude, reaching $-26.9 \pm 5.6 \%$ at $10^{-5} \mathrm{M}$ (Fig. 1C). Selective COX-2 inhibitors celecoxib and firocoxib did not induce relevant modifications of EFS-evoked contractions, whereas parecoxib caused a marked and concentration-dependent increase of the contractions, with a maximal effect of $+69.1 \pm 22.6 \%$ at $10^{-4} \mathrm{M}$ (Fig. 1D).

None of the studied NSAIDs, in the range of concentrations $10^{-9}-10^{-4} \mathrm{M}$, was able to affect the basal tone of the bronchial preparations (not shown).

Diclofenac, meloxicam, and parecoxib at the concentration of $10^{-5} \mathrm{M}$ potentiated the concentration-response curves of $\mathrm{ACh}$, while carprofen caused a minor leftward shift of the ACh curve (Fig. 2A-D). The pD2 values for ACh curves were significantly increased after 

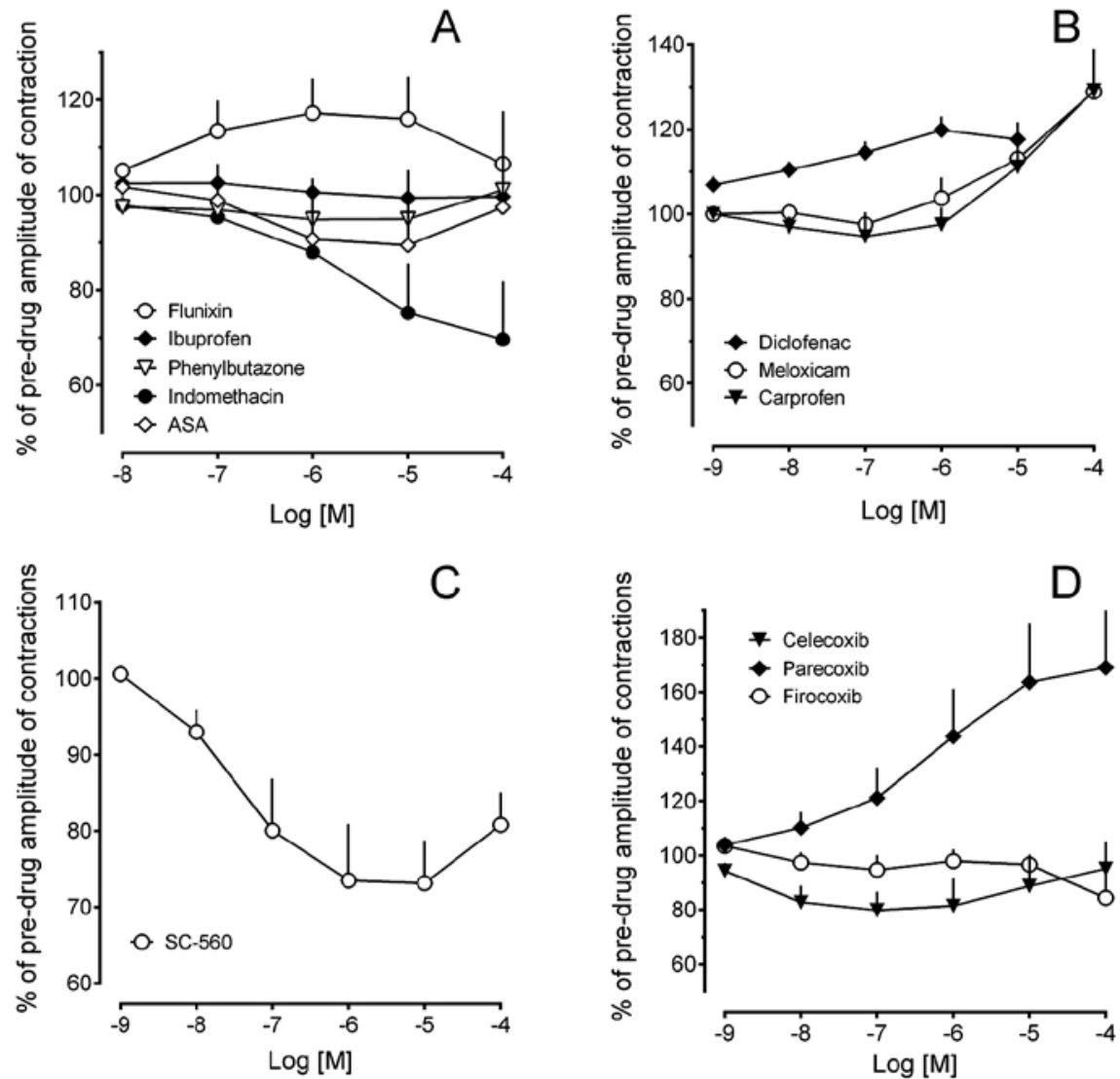

Fig. 1. Effects of nonselective cyclooxygenase (COX)-1/COX-2 inhibitors (A), preferential COX-2 inhibitors (B), selective COX-1 inhibitor (C), and selective COX-2 inhibitors (D), on electrical field-stimulated contractions of the horse's bronchial smooth muscle

incubation with a single $10^{-5} \mathrm{M}$ aliquot of diclofenac, meloxicam, and parecoxib (Table 1). On the contrary, diclofenac, meloxicam, carprofen and parecoxib were unable to potentiate the concentration-response curve of carbachol (not shown).

Both indomethacin and compound SC-560, up to $10^{-5} \mathrm{M}$, were devoid of measurable effects on the contraction plateau of bronchial smooth muscle induced by $10^{-5} \mathrm{M}$ carbachol (data not shown).

\section{Discussion}

At present, available data about the effects of NSAIDs on the motility of the respiratory tract are controversial, and their interpretation may be complex. Prostanoids exert variable effects on airway smooth muscle of different species. Prostaglandin $\mathrm{E}_{2}$ and prostaglandin $\mathrm{I}_{2}$ $\left(\mathrm{PGI}_{2}\right)$ relax the tracheal muscle of guinea pigs, whereas prostaglandin $\mathrm{F}_{2 \alpha}\left(\mathrm{PGF}_{2 \alpha}\right)$, prostaglandin $\mathrm{D}_{2}\left(\mathrm{PGD}_{2}\right)$ and thromboxane $\mathrm{A}_{2}\left(\mathrm{TXA}_{2}\right)$, induce a contraction (Hamberg et al. 1975); human bronchial muscle contracts under the effect of $\mathrm{PGF}_{2 \alpha}$, and is relaxed by prostaglandin $\mathrm{E}_{1}\left(\mathrm{PGE}_{1}\right)$, whereas $\mathrm{PGE}_{2}$ induces either contraction or relaxation (Gardiner 

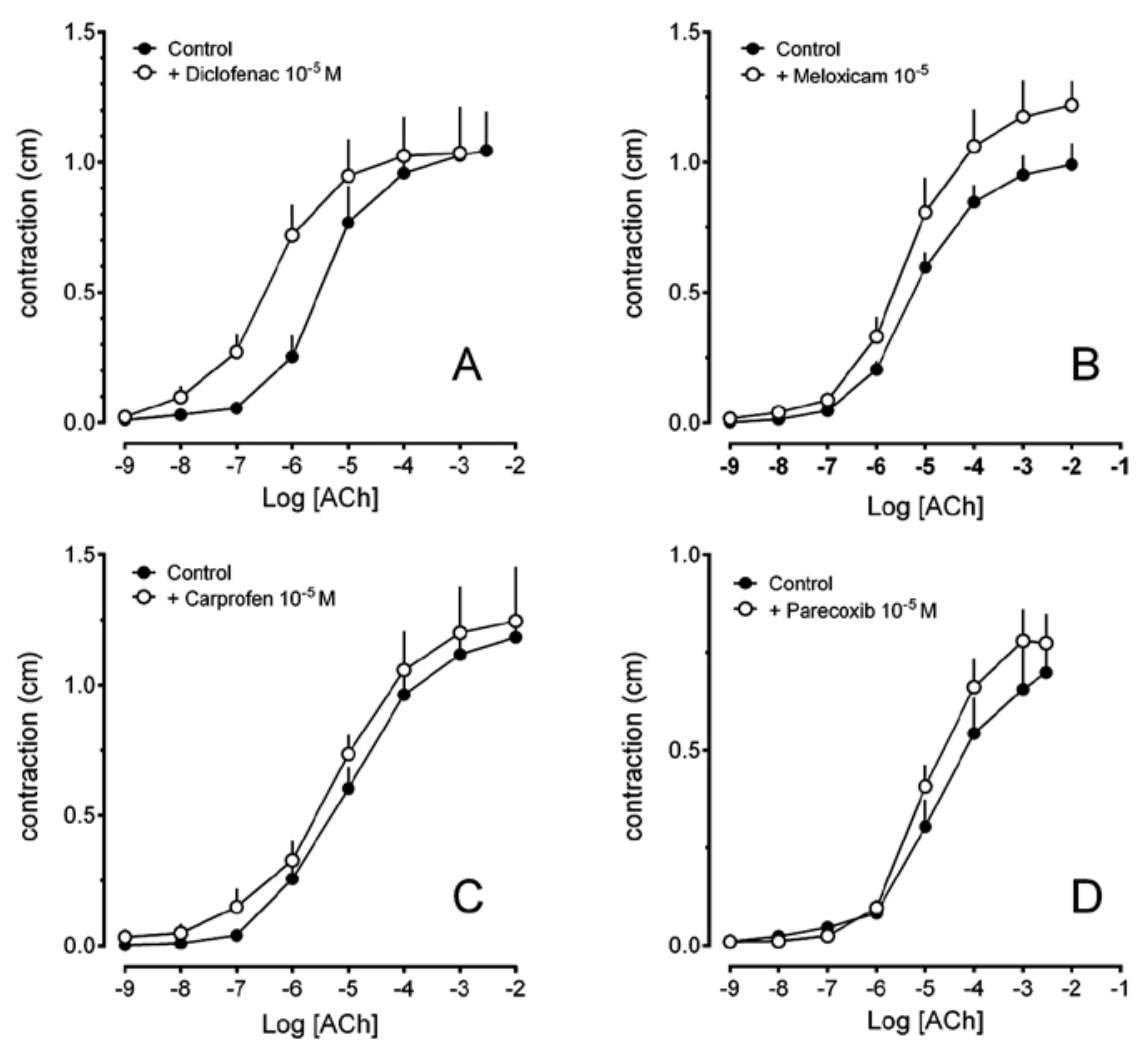

Fig. 2. Acetylcholine-induced contraction of the horse's bronchial smooth muscle in absence (black dots) and after the incubation with $10^{-5} \mathrm{M}$ diclofenac (A), meloxicam (B), carprofen (C), and parecoxib (D)

1975). Furthermore, $\mathrm{PGE}_{1}, \mathrm{PGE}_{2}$ and $\mathrm{PGF}_{2 \alpha}$ relax the smooth muscle of the cat trachea (Horton and Main 1965).

Orehek et al. showed that indomethacin and ASA, two NSAIDs with different chemical structures, reduced the basal tone of trachealis strips and enhanced the contractions caused

Table 1. Potency values (- $\log \mathrm{EC}_{50}$ ) calculated for concentration-response curves of acetylcholine in absence (Control) and after incubation with $10^{-5} \mathrm{M}$ diclofenac, meloxicam, carprofen, and parecoxib in the horse's bronchial smooth muscle.

\begin{tabular}{llll}
\hline \multicolumn{3}{c}{ Control } & \multicolumn{1}{c}{+ NSAID } \\
\hline Diclofenac & $5.46 \pm 0.11$ & $6.37 \pm 0.09^{*}$ & $P=0.0002$ \\
Meloxicam & $5.14 \pm 0.06$ & $5.53 \pm 0.08^{*}$ & $P=0.021$ \\
Carprofen & $5.06 \pm 0.12$ & $5.22 \pm 0.10$ & $P=0.38$ \\
Parecoxib & $4.56 \pm 0.05$ & $5.02 \pm 0.08^{*}$ & \\
\hline
\end{tabular}

* $P<0.05$ vs control

Data represent means \pm standard error of the mean from eight experiments by different agonists in guinea pigs (Orehek et al. 1973; Orehek et al. 1975). Similarly, carbachol-induced contraction of the tracheal muscle was increased by indomethacin in the dog (McGrogan and Daniel 1996). On the contrary, despite effectively preventing the prostanoid synthesis in the bronchial muscle of men, NSAIDs did not modify the tone or the agonist-mediated contraction of such smooth muscle (Brink et al. 1980; Haye-Legrand et al. 1986). Previous experiments conducted 
in vivo in humans yielded rather conflicting results, as although large doses of NSAIDs did not modify the bronchial tone in most asthmatic patients (Smith 1975; Bianco et al. 1985 ), in $4 \%$ of them a worsening of clinical signs occurred, and in $1 \%$ an amelioration was observed (Hanley 1986). The improvement is thought to be due to a depletion of prostanoids causing bronchoconstriction (Smith and Cuthbert 1976; Hanley 1986), whereas the worsening is likely caused by an increased conversion of arachidonic acid to spasmogenic leukotrienes by 5-lipooxygenase pathway (Christie et al. 1991; Parker et al. 2016). Why NSAIDs could induce contrasting effects in patients with the same disease remains to be clarified, even though an individual variability in the synthesis of prostanoids exerting opposite actions on the smooth muscle tone might be suspected. Collectively, previous evidence suggests that although COX enzymes and their products may be involved in the modulation of the airway motility, their role differ considerably based on species, airway portion, and experimental conditions.

The effects induced by NSAIDs observed in the present study suggest that the existence of a basal production of prostanoids modulating EFS-evoked contractions of isolated bronchi in the horse should be excluded. In fact, even though some of the studied NSAIDs caused a modification of the contraction amplitude, such effects do not seem related to the inhibition of prostanoid synthesis, since other drugs with similar COX-1/COX-2 selectivity were ineffective. Moreover, NSAIDs produced in some cases opposite effects on EFS-induced contractions: diclofenac, carprofen, meloxicam, and parecoxib caused an enhancement of the contractions, whereas indomethacin and SC-560 induced their decrease. No correlation between the chemical structure and effect was observed either, given that carprofen and parecoxib caused an increase of the contractions, whereas structurally similar NSAIDs ibuprofen, celecoxib, and firocoxib were ineffective. Indeed, although COX-2 selective inhibitors celecoxib and rofecoxib were proved to be safe in human patients with NSAIDsensitive asthma (Martin-Garcia et al. 2002; Martin-Garcia et al. 2003), two cases of severe bronchospasm following parecoxib administration were reported (Looney et al. 2005), suggesting that such adverse reactions may be caused by peculiar properties of the drug, rather than by the main mechanism of action.

The effects induced by NSAIDs on EFS-evoked contractions in our study are likely due to a modulation of $\mathrm{ACh}$ actions on the bronchial muscle of horses, since these contractions have been previously shown to be of fully cholinergic nature (Menozzi et al. 2014), and could involve either the release or the degradation of the neurotransmitter. An interaction by NSAIDs with post-synaptic receptors seems to be unlikely, since none of the studied drugs was able to alter the basal tone of the preparations. Even though it was proposed that prostanoids could affect the motility of the respiratory system by modulating neurotransmitter release (Shore et al. 1985; Zhao et al. 1994), and this might partially explain the difference between data obtained in vivo and in vitro, the results obtained in the present study suggest that this does not occur in isolated bronchi of horses. In accordance, indomethacin, meclofenamate or $\mathrm{PGE}_{2}$ were previously found unable to modify the ACh release in equine bronchi (Zhao et al. 1994).

A hypothesis for the increase of the phasic contractions induced by diclofenac, meloxicam, carprofen, and parecoxib could be that these drugs might inhibit acetylcholinesterase, thus enhancing the effects of endogenous ACh released by EFS. The effects of these NSAIDs on exogenous ACh seem to confirm this, since an increase of agonist potency was observed, whereas the concentration-response curves of carbachol, which is resistant to degradation by acetylcholinesterase, were not significantly shifted. Indeed, NSAIDs with different chemical structures were previously shown to inhibit the acetylcholinesterase activity (Goverdhan et al. 2012; Parmar et al. 2017). In those studies, however, celecoxib, ibuprofen, and ASA, which were ineffective in our experiments, also displayed an acetylcholinesterase inhibitor activity. Further studies will be needed to clarify the reason for these discrepancies. 
Indomethacin and SC-560 caused a reduction of neurogenic contractions of horse bronchi, and also this effect cannot be attributed to COX inhibition but could possibly involve an interference with ACh release. The lack of effect of both drugs on the basal tone or carbachol-induced contraction suggests that an interaction with post-synaptic receptors located on the smooth muscle is instead unlikely to be involved. Previous studies showed that the indomethacin-induced inhibition of the contraction of the guinea pig ileum induced by ACh or other agonists is unrelated to prostaglandin depletion (Aboulafia et al. 1976). It could be speculated that indomethacin or SC-560 might inhibit calcium voltagegated channels. In accordance with this hypothesis, Northover et al. (1971) found that indomethacin reduced the EFS-stimulated contractions of stomach in the guinea pig by decreasing the calcium uptake into muscle cells. This NSAID also seems to inhibit the motility of human uterus by blocking calcium ion currents (Sawdy et al. 1998).

The results obtained in this study may also have some interesting clinical implications. Among the NSAIDs commonly employed in horses, phenylbutazone and firocoxib could be considered safe with regard to the risk of causing adverse reactions due to bronchoconstriction. On the contrary, the use of meloxicam and carprofen might require some caution, especially when treating horses with bronchial hyper-reactivity or obstructive respiratory diseases like RAO, since these NSAIDs tend to enhance cholinergic contractions of isolated bronchi. Moreover, the perioperative use of these NSAIDs could also increase the risk of inducing respiratory side effects, given that horses seem to be particularly susceptible to respiratory stress connected to general anaesthesia (Grosenbaugh et al. 1998).

Indomethacin was shown to inhibit EFS-induced contractions of the horse bronchi, and thus might be potentially useful in different clinical conditions by increasing pulmonary ventilation. However, its safety profile in horses seems to be lower (Roberts 1982) with respect to other NSAIDs, such as phenylbutazone or firocoxib, and thus a future clinical use of this drug in equine practice is rather unlikely.

\section{Acknowledgements}

The authors wish to thank the workers of the "Titbit" slaughterhouse in Bagnolo in Piano (RE), Italy, for their precious assistance. This study was supported by a grant from the University of Parma (FIL 2014).

\section{References}

Aboulafia J, Mendes GB, Miyamoto ME, Paiva AC, Paiva TB 1976: Effect of indomethacin and prostaglandin on the smooth muscle contracting activity of angiotensin and other agonists. Br J Pharmacol 58: 223-228

Atukorala I, Hunter DJ 2013: Valdecoxib: the rise and fall of a COX-2 inhibitor. Expert Opin Pharmacother 14: 1077-1086

Bianco S, Robuschi M, Damonte C, Simone P, Vaghi A, Pasargiklian M 1985: Bronchial response to nonsteroidal anti-inflammatory drugs in asthmatic patients. Prog Biochem Pharmacol 20: 132-142

Bombardier C, Laine L, Reicin A, Shapiro D, Burgos-Vargas R, Davis B, Day R, Ferraz MB, Hawkey CJ, Hochberg MC, Kvien TK, Schnitzer TJ, Group VS 2000: Comparison of upper gastrointestinal toxicity of rofecoxib and naproxen in patients with rheumatoid arthritis. VIGOR Study Group. N Engl J Med 343: $1520-1528$

Brideau C, Van Staden C, Chan CC 2001: In vitro effects of cyclooxygenase inhibitors in whole blood of horses, dogs, and cats. Am J Vet Res 62: 1755-1760

Brink C, Grimaud C, Guillot C, Orehek J 1980: The interaction between indomethacin and contractile agents on human isolated airway muscle. Br J Pharmacol 69: 383-388

Christie PE, Tagari P, Ford-Hutchinson AW, Charlesson S, Chee P, Arm JP, Lee TH 1991: Urinary leukotriene E4 concentrations increase after aspirin challenge in aspirin-sensitive asthmatic subjects. Am Rev Respir Dis 143: $1025-1029$

Dabareiner RM, White NA 1995: Large colon impaction in horses: 147 cases (1985-1991). J Am Vet Med Assoc 206: $679-685$

Douglas JS, Brink C 1987: Airway smooth muscle and disease workshop: histamine and prostanoids. Am Rev Respir Dis 136: S21-S24 
Futaki N, Takahashi S, Yokoyama M, Arai I, Higuchi S, Otomo S 1994: NS-398, a new anti-inflammatory agent, selectively inhibits prostaglandin $\mathrm{G} / \mathrm{H}$ synthase/cyclooxygenase (COX-2) activity in vitro. Prostaglandins 47: 55-59

Gardiner PJ 1975: The effects of some natural prostaglandins on isolated human circular bronchial muscle. Prostaglandins 10: 607-616

Goverdhan P, Sravanthi A, Mamatha T 2012: Neuroprotective effects of meloxicam and selegiline in scopolamineinduced cognitive impairment and oxidative stress. Int J Alzheimers Dis 2012: 974013

Gray PR, Derksen FJ, Broadstone RV, Robinson NE, Peters-Golden M 1992: Decreased airway mucosal prostaglandin E2 production during airway obstruction in an animal model of asthma. Am Rev Respir Dis 146: $586-591$

Grosenbaugh DA, Muir WW 1998: Cardiorespiratory effects of sevoflurane, isoflurane, and halothane anesthesia in horses. Am J Vet Res 59: 101-106

Hamberg M, Hedqvist P, Strandberg K, Svensson J, Samuelsson B 1975: Prostaglandin endoperoxides IV. Effects on smooth muscle. Life Sci 16: 451-462

Hanley SP 1986: Prostaglandins and the lung. Lung 164: 65-77

Harrington LS, Lucas R, McMaster SK, Moreno L, Scadding G, Warner TD, Mitchell JA 2008: COX-1, and not COX-2 activity, regulates airway function: relevance to aspirin-sensitive asthma. FASEB J 22: 4005-4010

Haye-Legrand I, Cerrina J, Raffestin B, Labat C, Boullet C, Bayol A, Benveniste J, Brink C 1986: Histamine contraction of isolated human airway muscle preparations: role of prostaglandins. J Pharmacol Exp Ther 239: 536-541

Horton EW, Main IH 1965: A Comparison of the actions of prostaglandins F2-alpha and E1 on smooth muscle. Br J Pharmacol Chemother 24: 470-476

Kargman S, Charleson S, Cartwright M, Frank J, Riendeau D, Mancini J, Evans J, O’Neill G 1996: Characterization of prostaglandin $\mathrm{G} / \mathrm{H}$ synthase 1 and 2 in rat, dog, monkey, and human gastrointestinal tracts. Gastroenterology 111: $445-454$

Looney Y, O'Shea A, O’Dwyer R 2005: Severe bronchospasm after parenteral parecoxib: cyclooxygenase-2 inhibitors: not the answer yet. Anesthesiology 102: 473-475

MacAllister CG, Morgan SJ, Borne AT, Pollet RA 1993: Comparison of adverse effects of phenylbutazone, flunixin meglumine, and ketoprofen in horses. J Am Vet Med Assoc 202: 71-77

MacKay RJ, French TW, Nguyen HT, Mayhew IG 1983: Effects of large doses of phenylbutazone administration to horses. Am J Vet Res 44: 774-780

Martin-Garcia C, Hinojosa M, Berges P, Camacho E, Garcia-Rodriguez R, Alfaya T 2003: Celecoxib, a highly selective COX-2 inhibitor, is safe in aspirin-induced asthma patients. J Investig Allergol Clin Immunol 13: 20-25

Martin-Garcia C, Hinojosa M, Berges P, Camacho E, Garcia-Rodriguez R, Alfaya T, Iscar A 2002: Safety of a cyclooxygenase-2 inhibitor in patients with aspirin-sensitive asthma. Chest 121: 1812-1817

McGrogan I, Daniel EE 1996: Release and actions of inhibitory prostaglandins from canine tracheal epithelium. Can J Physiol Pharmacol 74: 1055-1060

Menozzi A, Pozzoli C, Poli E, Delvescovo B, Serventi P, Bertini S 2014: Pharmacological characterization of muscarinic receptors in the contractions of isolated bronchi in the horse. J Vet Pharmacol Ther 37: 325-331

Northover BJ 1971: Mechanism of the inhibitory action of indomethacin on smooth muscle. Br J Pharmacol 41: 540-551

Orehek J, Douglas JS, Bouhuys A 1975: Contractile responses of the guinea-pig trachea in vitro: modification by prostaglandin synthesis-inhibiting drugs. J Pharmacol Exp Ther 194: 554-564

Orehek J, Douglas JS, Lewis AJ, Bouhuys A 1973: Prostaglandin regulation of airway smooth muscle tone. Nat New Biol 245: 84-85

Parker AR, Ayars AG, Altman MC, Henderson WR, Jr. 2016: Lipid mediators in aspirin-exacerbated respiratory disease. Immunol Allergy Clin North Am 36: 749-763

Parmar HS, Assaiya A, Agrawal R, Tiwari S, Mufti I, Jain N, Manivannan E, Banerjee T, Kumar A 2017: Inhibition of $A \beta(1-42)$ oligomerization, fibrillization and acetylcholinesterase activity by some anti-inflammatory drugs: an in vitro study. Antiinflamm Antiallergy Agents Med Chem 15: 191-203

Roberts MC 1982: Adverse effects of indomethacin in the horse. J Vet Pharmacol Ther 5: 83-86

Sawdy R, Knock GA, Bennett PR, Poston L, Aaronson PI 1998: Effect of nimesulide and indomethacin on contractility and the $\mathrm{Ca}^{2+}$ channel current in myometrial smooth muscle from pregnant women. Br J Pharmacol 125: $1212-1217$

Shore SA, Powell WS, Martin JG 1985: Endogenous prostaglandins modulate histamine-induced contraction in canine tracheal smooth muscle. J Appl Physiol 58: 859-868

Smith AP 1975: Effect of indomethacin in asthma: evidence against a role for prostaglandins in its pathogenesis. Br J Clin Pharmacol 2: 307-309

Smith AP, Cuthbert MF 1976: The response of normal and asthmatic subjects to prostaglandins E2 and F2alpha by different routes, and their significance in asthma. Adv Prostaglandin Thromboxane Res 1: 449-459 
Steinhoff MS, von Mentzer B, Geppetti P, Pothoulakis C, Bunnett NW 2014: Tachykinins and their receptors: contributions to physiological control and the mechanisms of disease. Physiol Rev 94: 265-301

Xie WL, Chipman JG, Robertson DL, Erikson RL, Simmons DL 1991: Expression of a mitogen-responsive gene encoding prostaglandin synthase is regulated by mRNA splicing. Proc Natl Acad Sci USA 88: 2692-2696

Zhao WW, Robinson NE, Yu MF 1994: PGE2 inhibits acetylcholine release from cholinergic nerves in canine but not equine airways. Prostaglandins Leukot Essent Fatty Acids 51: 347-355

Zimmermann KC, Sarbia M, Schror K, Weber AA 1998: Constitutive cyclooxygenase-2 expression in healthy human and rabbit gastric mucosa. Mol Pharmacol 54: 536-540 